\title{
Exercise strategy for avoiding disability improving life expectancy and reducing healthcare costs
}

\author{
M Provan ${ }^{1 *}$ and AM Mander ${ }^{2}$ \\ ${ }^{1}$ John Radcliffe hospital, Oxford University Hospitals NHS Foundation Trust, UK \\ ${ }^{2}$ Faculty of Medical and Human Sciences, Manchester University, Institute of Cancer Studies, Manchester, UK
}

\section{Definition of exercise}

NOUN- "Activity requiring physical effort, carried out to sustain or improve health and fitness" [1].

\section{Introduction}

We live in a world of contrast.

On the one hand we are suffering from a rapidly increasing obesity epidemic with fast food options available at every corner and increasing rates of co-morbidities and other health issues, but on the other hand, thanks to social media trends in our ever-growing technological world, the obsession over body image seems never to have be as dominant, with individuals appearing more body conscious than ever.

So rather like the famous children book "Fattypuffs and Thinifers" [2]. The population is split into those who are overweight and challenging to the NHS/healthcare systems, whether it be that they are uneducated or unable to help themselves and those who obsess over their body image in a social media driven reality where they are constantly looking for the next exercise phenomena or new diet craze.

Many people in society lead very sedentary lifestyles; sitting down too much i.e. watching television for hours on end, playing computer games and so on, sitting down of course cannot be avoided but prolonged periods of inactivity can be adjusted. Working at a standing desk, timers to get up and move every hour and walking meetings are just some of examples of how we could change to encourage more movement.

One just has to look at the multitude of afternoon TV adverts for reclining chairs to observe the insidious development of a sedentary lifestyle is usually progressive and leads to the onset of illness. It is perhaps not inappropriate that the many adverts for reclining chairs, are often followed by those for funeral plans.!

It is vital to keep muscles strong, avoiding sarcopenia, and the bones and joints in good health [3]. Can we prevent sarcopenia and improve longevity and healthy ageing by simply interacting with each other to motivate ourselves and others around us to get up off the sofa and complete those 10,000 steps or achieve our fitness targets?

Who should be responsible and how can they motivate the average person to engage in physical exercise and incorporate it as part of their daily routine and help disability avoidance? Should responsibility lie with the NHS, the government, councils, schools/educational groups?

Could group exercise be a solution.

\section{History of group exercise}

Group exercise dates back to ancient Greece. Public places were devoted to training venues for athletes. The Greeks regarded gymnastic excellence as a noble and godly pursuit and it was included as a part of a complete education with a focus on self-discipline. Gymnasia became the centre of the community, skilled athletes attained an elevated status and devoted their lives to becoming proficient in exercise.

Through the 1800s exercise was used to "mould better citizens" and enhance the body, reforms were made in regard to exercise and systems were in place to improve overall fitness of athletes. It was during the 19th century that the importance of physical exercise for all, men women and children alike, attained a position in everyday life.

Totalitarian regimes used group gymnastics as a way to promote their ideologies. Physical fitness was at the core and governments financed the construction of sports and wellness facilities.

The Cold War saw the further evolution of physical fitness in the US and Soviet Union. It was said that communist dominance came from superior sports and fitness programmes and as a result Americans were urged to prioritise their physical fitness. Fitness was clearly described as a "matter of achieving an optimum state of well-being" that required exercise from both young and old. This focus on fitness also opened the doors for female athletes in both the US and the USSR to become more prominent as contenders in the Olympics.

The 1900s saw the rise of non-organised health-orientated exercise. By the 70s we were performing aerobics to music and people began engaging in individualistic physical activity. The focus was no longer on public health but individuals' desire to have fun, keep fit and attain self-improvement [4].

Recent developments in social media provide both positive and negative influences which can drive people to want to change their bodies in terms of plastic surgery and get overly obsessive with the way they perceive themselves which can lead to anorexia nervosa and bulimia. However social media can also have a majorly positive impact

*Correspondence to: Maisy Provan, Bsc Hons Physiotherapy, MCSP, John Radcliffe hospital, Oxford University Hospitals NHS Foundation Trust, UK, E-mail: maisy.provan@ntlworld.com

Key words: exercise, disability, sarcopenia, life expectancy, reducing healthcare costs

Received: October 15, 2018; Accepted: October 25, 2018; Published: October 29,2018 
in terms of motivation to exercise, it can also engage people and push them to try new forms of workout or activity.

\section{Evidence of Tai Chi/ Group exercise at work}

From antiquity have Asian communities held the secret to group exercise for longer than we have in western society? For years now, Asian populations have taken time out of their days to come together to engage in group exercise in the forms of tai chi and yoga. The overall longevity and greater life expectancy in these countries could be a reflection of just this. So, is group exercise one answer to living longer?

Tai Chi was developed as an ancient Chinese martial art and today is widely revered for its health benefits. It incorporates the concepts of ying and yang which represent two opposing, but yet complementary forces described in traditional Chinese Medicine. By balancing these two opposing forces the individual should supposedly achieve good health. The movement series in tai chi is designed to strengthen and stretch the body to improve the flow of blood and other fluids throughout the body, while simultaneously enhancing balance, proprioception and body awareness. Tai Chi has been adopted as a popular activity in the west, taught as a series of slow, gentle, low-impact movements that integrate the mind, with the physical activity using breath as a focal point. This, in turn, pushes the individual to achieve a sense of inner peace and well-being.

Ta Chi has been shown to play in a role in improving many chronic conditions; Osteoporosis, Parkinson's cardiovascular disease, and rheumatoid arthritis to name a few [5]. It has also been linked with positive effects on menopause and dementia, however there is a role for further research to be carried out in these areas especially with regard to sarcopenia.

\section{The evolution of group exercise- Where are we now?}

Moving through the 21st century we have observed a change in the pursuit of fitness. The ways of exercising have evolved, who ever thought we would have developed from Greek gymnasts to getting together in community halls to take part in Zumba classes.

Many influential people have shaped the way we perceive exercise and influenced the way we do.

In 1968 Cooper [6] introduced the concept of aerobic exercise and advocated a philosophy that shifted away from disease treatment to one of disease prevention. "It is easier to maintain good health through proper exercise, diet and emotional balance than it is to regain it once it is lost,". Goldberg [6] developed the idea for spin classes in the late 1980s, and finally in 1963 Casani [6] developed the Swiss Ball, originally just used by physiotherapists has since transitioned from the clinical environment to mainstream fitness settings [6].

Gyms have turned from simple places to exercise to fitness centres and health clubs with social areas and cafes, classes and instructors, ranging from yoga to step, aerobics to spin and various other danceinspired exercise classes. Group exercise has ventured even further from yoga in the park to local bootcamps even latin or ballroom dancing. Going into your local gym you can now observe how fitness has become commercialized, some are heavy influenced by social media and workout to reach aesthetic ideals influenced by the development of our social media generation, working, for example, to develop muscle mass and lift extremely heavy weights.

Technology has also had a massive impact on the way we exercise, whether it's tracking fitness or using devices to support and aid workouts; pedometers, GPS, heart rate monitors and smartphone apps to quantify/ monitor exercise efforts [4]. The influence of technology has led to people being able to have actual data for the exercise they have done or in some cases haven't, it allows people to set personal goals, specific to what they want to achieve.

We can also see the development of functional training i.e. Crossfit, which has its basis on developing all-round athletes rather than simply focusing on a single area of fitness [7]. Functional based training has been used by physiotherapists for years when developing rehabilitation programmes with patients.

Recent research developments have identified categories of training, fitness regimes and types of activity to be undertaken. Incorporating exercise into daily routines has been shown to be better than rationing over the week. Fitting activity into daily routines is also very beneficial i.e. taking the stairs rather than the lift, trying cycling/walk to work rather than using the car.

\section{Benefits of group exercise- Health wealth and happiness}

So why work out as a group when you can do it on your own, humans or most of us are social animals.

Group exercise brings with it a sense of motivation and camaraderie and sometimes a competitive element. It should be fun, a great social activity allowing interaction with others whilst improving physical fitness. Group activity in a class environment involves advice from a qualified instructor which is beneficial, especially if you are new to exercise.

Group exercise should be enjoyable and good fun due to its diversity and a wide variety of styles, formats and levels, ranging from beginner to advance with different workout styles. People simply have to show up with a positive attitude, participate, and most importantly enjoy. It is shown that people stay more interested due to the social atmosphere and interaction with others making the time go by much quicker.

Exercising with others can also help to improve healthy lifestyle behaviours and facilitate changes to diets and social habits [8]. Group exercise provides many social benefits, but more research is needed in this area and as further social and healthcare benefits are proven then resourcing should be easier to facilitate.

There are many newly recognized health benefits of activity in adults; apart from the well-established, including improving sleep, controlling weight (BMI), reducing stress and improving measurements of quality-of-life [3].

Exercise activity dramatically reduces the chances of the following serious and life-threatening illnesses which are potent causes of serious chronic disability:-

- type II diabetes by $40 \%$

- cardiovascular disease by $35 \%$

- falls depression and dementia by $30 \%$

- joint and back pain by $25 \%$

- the variety of malignancies, including colonic and breast cancer $-20 \%$ [3]

Weight bearing exercises that should be utilized to build strength, avoiding osteoporosis, sarcopenia and improve bone density include; formal gymnasium activities, yoga, dance, resistance training and Tai Chi. Dance is especially good as it helps to build strength whilst 
improving and maintaining balance, which is very important in ageing to avoid potential fractures and the risks of injury [3].

\section{Barriers and obstacles to exercise}

Many People recognize that exercise is good for them and know that they should participate in a form of exercise regularly, however, many find barriers or obstacles to prevent them from exercising. There are real barriers such as time constraints, commitments from work or children, lack of finance or facilitates can also be a major issue. With the appropriate information, motivation and education it would be simple enough to go for a jog or a run or complete body weight exercises where no equipment is required, however going with someone or in a group can be more motivating, i.e. by joining a running club or getting together with friends to try a new sport or activity. In lots of communities there are local group classes going on, sometimes people just don't know how to find out about them or are often afraid to try something new.

We could also link lack of education about exercise, nutrition and healthy lifestyle as a cultural obstacle to exercise, being brought up in a negative and deprived culture with smoking, alcohol and drugs is a major disincentive to a healthy lifestyle. Some children grow up in environments where they are unable to play with friends or do sport in schools and do not have education about healthy eating and lifestyle choices, therefore the government needs to look into more educational programmes avoiding exclusion and causes of disability, in achieving healthy lifestyles.

There is an overwhelming case for providing free state funded exercise programmes to give people an opportunity to try a new sport or activity to engage them initially. Schools should encourage children playing together and completing physical activity in a social environment with enjoyment being the focus rather than exercise. By engaging children from a young age will be more cost effectiveness in the long term.

Sometimes it's the simple initiation of beginning to exercise and fitting it into daily routines, healthcare professionals could lead and give more guidance as role models to combat the obesity and health epidemics and kick start the future to a healthier active society. Exercise needs to be made to seem appealing again like it did when we were children, we need to encourage the population to make time to exercise and overcome obstacles of boredom, solitude and inactivity and fear of where and how to begin. However, we also branch into discussion over which health professionals should be leading this drive as many healthcare professionals are often poor role models to society in terms of healthy lifestyle choices.

Physiotherapy, health and well-being services therefore need to be extended to all for longer term benefits to cost of healthcare to the NHS and to provide this well needed education about the importance of exercise, activity and healthy lifestyle choices.

\section{Improving quality of life}

Evidence suggests that group exercise improves quality of life and reduces stress compared with those who exercise alone [9]. Not only do we benefit ourselves when exercising with other through motivation we help to motivate others around us. It allows people to come together and helps to support the concept of mental, physical and emotional approach to health. Group exercise can improve quality of life through improving mental state therefore helping people who suffer from anxiety and depression.
Along with all the benefits generally exercising can have for people with OA, COPD, diabetes, menopause and can help those with OA and work towards aiding falls prevention in the elder population.

Although we have an increasing life expectancy rate, how much of the increase in life expectancy will be disability free? Who wants to live longer if they cannot enjoy their time and be physically disadvantaged? Educating people of the importance of exercise and healthy lifestyle choices will increase the disability free life expectancy, improving overall quality of life, whilst at the same time dramatically reducing overall health care costs, improving the national wealth. So, health wealth and happiness may well make the country and health service wealthier.

\section{Conclusion}

"I get by with a little help from my friends" John Lennon [10].

Looking into the future how can we motivate people to keep active, is group exercise an answer. How can this be facilitated to ensure and increase in disability-free life? Multi-disciplinary teams need to work together to prevent the development of chronic diseases through exercise and daily fitness [6]. Aiming to engage people by incorporating exercise into daily work routines. We should learn something from other cultures, making time to carry out group exercise in work place and community settings.

Showing people, the benefits of activity and give them the opportunity to feel young again and engage in physical activity with others.

Health, wealth and happiness, exercise may not make you wealthy, but it will make you healthy and happy.

\section{References}

1. Oxford dictionary (2010) Publisher: Oxford Univeristy Press: Oxford.

2. Fattypuffs and Thinifers (1941) Translation of the French children's book Patapoufs et Filifers originally written in 1930 by André Maurois. It concerns the imaginary underground land of the fat and congenial Fattypuffs and the thin and irritable Thinifers, which is visited by the Double brothers, the plump Edmund and the thin Terry. Fattypuffs and Thinifers do not mix, and their respective countries are on the verge of war when Edmund and Terry make their visit.

3. UK chief medical officers' guidelines (2011) Start active, Stay active.

4. Wikipedia (2018) Fitness Culture.

5. Paul G Shekell (2014) Evidence Map of Tai Chi.

6. Lance C Dalleck (2017) From Ancient Greece to Zumba: 50 Events, People and Trends That Have Shaped the History of Fitness (Part 2).

7. Crossfit (2018) What is CrossFit?

8. Dolan S (2018) American College of Sports Medicine (ACSM).

9. American Osteopathic Association (2017) Group exercise improves quality of life, reduces stress far more than individual work outs. Science Daily.

10. Lennon J (1967) With a Little help from my friends". The Beatles.

Copyright: (C2018 Provan M. This is an open-access article distributed under the terms of the Creative Commons Attribution License, which permits unrestricted use, distribution, and reproduction in any medium, provided the original author and source are credited. 